\title{
INTERNATIONAL COSMIC RAY CONFERENCE
}

\author{
MEXICO MEETING
}

$\mathrm{S}^{\mathrm{i}}$ NCE the War, a series of international conferences on cosmic rays has been organized by the Cosmic Ray Commission of the International Union of Pure and Applied Physics. These conferences have been arranged at two-yearly intervals, the first being held in Cracow in 1947, the second in Como in 1949, the third in Bombay in 1951, and the fourth in Bagnères de Bigorre in 1953. This last conference was almost exclusively concerned with the fundamental particle aspect of cosmic ray physics, although there was a small parallel meeting on time-variations of the cosmic ray intensity.

In contrast to the Bagnères de Bigorre meeting the topics chosen for the 1955 conference were the composition and energy spectrum of the primary radiation, geomagnetic effects, variations of the intensity with time and, finally, the problem of the origin of the radiation.

The conference was held in Guanajuato, a beautiful old Colonial town some 300 miles north of Mexico City, during September 5-13, and was made possible by generous grants from the Mexican Government and from Unesco. The meetings were held at the University of Guanajuato, where facilities were kindly provided by the rector, Lic. Antonio Torres Gomez. The detailed arrangements for the conference were made by Prof. M. S. Vallarta and the president of the Cosmic Ray Commission, Prof. P. M. S. Blackett. In addition to some sixty cosmicray physicists working on the topics covered by the conference, a number of astronomers (Brown, Chandrasekhar, Gold, Kiepenheuer, Oort, Schluter, Unsöld and Van de Hulst) also attended, and the meeting provided a valuable opportunity for joint discussion of problems of common interest.

The first day of the conference was devoted to papers and discussion on the composition and flux of the primary radiation and on the influence of the earth's magnetic field on the intensity of the radiation. Some recent work by the Iowa group was described which confirms the presence of a soft radiation in the auroral zone at altitudes greater than $50 \mathrm{~km}$. It is believed that this radiation consists of electrons of rather low energy, which may belong to the high-energy tail of the auroral particle spectrum. The question of the abundance of lithium, beryllium and boron in the primary flux was discussed at some length, but the proportion of these elements present is still in doubt. The results of a recent latitude survey carried out by the Chicago and Ottawa groups using shipborne neutron monitors and meson telescopes were reported. The position of the geomagnetic equator deduced from these measurements appears to differ from that corresponding to the dipole field deduced from magnetic measurements, leading to an apparent discrepancy of as much as $7^{\circ}$ in the geomagnetic latitudes of some stations.

The energy spectrum of the primary radiation was discussed on the second day. Data were presented. by a number of workers on the low-energy end of the spectrum. In particular, the position of the knee in the intensity versus latitude curve was discussed; and evidence was presented showing that the position of the knee changes with time, being virtually absent at sunspot minimum, so that the intensity of the low-energy cosmic radiation was greatly increased at this time. The spectrum at high energies is deduced from measurements on large air showers, and several arrangements which are at present being used for the study of these showers were described. The radial density distribution of particles in showers of energy in the region of $10^{17} \mathrm{eV}$. has been investigated at Harwell and also by Russian workers using large counter arrays; some evidence was presented by the Harwell group for the existence of showers of energy $10^{19} \mathrm{eV}$. Some preliminary results were reported by the group from the Massachusetts Institute of Technology, which has recently completed a shower detector consisting of twenty large scintillator units. There was some discussion on the estimation of the primary-particle energy responsible for a given shower size, and there seemed to be general agreement among the different workers that the primary energy is given by the total number of particles in the shower multiplied by a factor of about $10^{10} \mathrm{eV}$.

The third and fourth days of the conference were taken up with the reporting and discussion of time variations of the cosmic-ray intensity. This session began with a discussion of meteorological effects on the sea-level intensity, in particular the relation between the intensity and atmospheric temperature. Then followed a series of contributions on the relation between the intensity and solar and geomagnetic activity. There were papers on cosmic-ray production associated with solar flares, intensity changes at the time of magnetic disturbances and the world-wide variation in the cosmic-ray intensity over the past solar cycle as recorded by the Carnegie Institution of Washington at a series of stations which have been in operation since 1936. Data from these stations show a variation in intensity during the solar cycle of about 4 per cent, maximum intensity occurring at the time of sunspot minimum. Discussion of the causes of these variations of intensity was mainly in terms of the possible effects of ejected solar material carrying magnetic fields out into interplanetary space, although there was no general agreement as to the precise way in which these fields produced the observed variations.

The fourth day was devoted to papers and"discussion on the solar daily variation and its behaviour in relation to solar and geomagnetic activity, the sideroal daily variation and measurements on the isotropy of the high-energy particles producing large air showers. The anisotropy of the primary radiation in relation to the Sun, as evidenced by the presence of a solar daily variation, is well established; but as yet there is no general agreement on the existence of a sidereal daily variation. A new approach to the problem of the isotropy of the primary radiation at high energies lies in the measurement of the directions of arrival of large air showers, using techniques permitting an angular resolution of $5^{\circ}$ or so. Some preliminary results of measurements of this kind were reported by the groups from the Massachusetts Institute of Technology and from the Imperial College of Science and Technology, London; but no definite anisotropy has so far been established with certainty. 
The remaining time at the conference was allotted to contributions on theories of the origin of the primary radiation. This session began with introductory papers on the properties of the galaxy, the interstellar gas and the solar atmosphere. Prof. J. H. Oort described some recent work at Leyden confirming earlier results by Russian workers on the polarization of the light from the Crab nebula. The mean polarization over the central region of the nebula is 17 per cent; but there are indications that where a limited volume of the nebula can be isolated the polarization may be complete. Oort pointed out that it is possible to account for both the polarization at optical wavelengths and the observed intensity of radio noise if it is assumed that the emission is due to radiation from electrons of energy $\sim 10^{11} \mathrm{eV}$. spiralling in a magnetic field of $10^{-3}$ gauss. If this interpretation is correct, it opens up the interesting possibility that the Crab nebula may be a source of part of the cosmic radiation. The papers and discussion on the origin of the radiation left little room for doubt, however, that this problem still remains largely unsolved and is likely to feature as a subject for discussion at perhaps more than one cosmic-ray conference in the future.

The conference lasted for six days, and it will be appreciated that in a report of this length it has only been possible to mention a few of the many important contributions, selected in order to illustrate present trends in these fields.

\section{SECOND INTERNATIONAL SEAWEED SYMPOSIUM}

$\mathrm{F}$

OLLOWING the suggestion put forward at the first International Seaweed Symposium, held in Edinburgh in 1952 (see Nature, 170, 478; 1952), the Norwegian Institute of Seaweed Research (Norsk institutt for tang-og tareforskning) arranged the second such symposium in Trondheim during July 1416, when about $a$ hundred and twenty scientific workers and industria]ists from all over the world attended. In his opening address, Prof. Trygfe Braarud, chairman of the Board of Management of the Norwegian Institute of Seaweed Research, welcomed the delegates to Norway.

At the first session, dealing with "Algal Chemistry", Dr. E. I. Pereival (Scotland) opened the proceedings with a paper on the polysaccharide sulphuric acid ester of the green alga, Cladophora rupestris, hydrolysis of which revealed L-arabinose, D-galactose, $\mathrm{D}$-xylose, L-rhamnose and $\mathrm{D}$-glucose, this being the first reported occurrence of arabinose in a seaweed polysaccharide; attempts to elucidate the structure of this complex polysaccharide by standard pro. cedures were described. Dr. E. T. Dewar (Scotland) discussed the preparation, estimation and use of sodium laminarin sulphate as a heparin-substitute for preventing blood from clotting. The production of antibiotic substances by seaweeds was the subject of a paper by Prof. C. G. C. Chesters (England), in which certain littoral and sublittoral seaweeds have been examined for bacteriostatic activity; Polysiphonia fastigiata and Halidrys siliquosa are particularly good sources, and there is evidence of seasonal variation of antibiotic substances. The distribution of microbiological growth factors related to vitamin $B_{12}$, folic acid, folinic acid, niacin and pantothenic acid in the Rhodophyceae, Phaeophyceae, Chlorophyceae and Heterokontae was discussed by Prof. H. Lundin (Sweden) in a paper with L.-E. Ericson. The red and green algae generally contain higher amounts of vitamins than the brown algae, the maximum $\mathrm{B}_{12}$ content being possessed by Vaucheria dichotoma $(2 \cdot 8 \mu \mathrm{gm}$./gm. dry weight). The origin of vitamin $B_{12}$ factors is believed to be the bacteria living epiphytically on the plant, the algae merely concentrating and storing the vitamin but not synthesizing it. The carotene and carotenoid content of seaweeds was dealt with in two papers (by A. Haug and B. Larsen, and S. Liaaen and N. A. Sørensen). Mr. Haug (Norway) discussed the carotene content of Pelvetia canaliculata, Fucus vesiculosus, $F$. serratus and Rhodymenia palmata, the quantity in the fresh algae being generally much higher than in the dried materials and commercial seaweed meals; drying and storing conditions are important factors in carotene breakdown. Prof. Sørensen (Norway) dealt with the carotenoids of fresh $F$. vesiculosus and conditions leading to the formation of zeaxanthin; it is now believed that this pigment is formed from violaxanthin, and not fucoxanthin as originally suggested. Low-molecular carbohydrates were reviewed by Dr. B. Lindberg (Sweden), who has shown that the mono- and di- $\beta$-D-glucosides of mannitol are characteristic of many Phaeophyceae, while $P$. canaliculata also contains the corresponding glucosides of D-volemitol; brown algae possess 'laminitol', a $C$-methyl inositol. The glyceric acid-mannose glycoside of red algae is proved to be 2-D-glyceric acid $\alpha$-D-mannopyranoside, while two recently isolated glycosides are floridoside- $\alpha$-mannoside and 'isofloridoside' (1-glycerol- $\alpha$-D-galactopyranoside). The taxonomical value of these compounds was discussed.

Dr. F. T. Haxo (United States), in a paper with C. O hEochdha, reviewed the constituents of Porphyridium cruentum, a unicellular red alga which grows well but not prolifically in mineral-enriched seawater. The culture medium becomes viscous due to the presence of extra-cellular mucilage, which consists in part of a sulphated polysaccharide containing glucose, galactose, xylose and a uronic acid. Pigments include chlorophyll $a$, $\beta$-carotene, zeaxanthin and possibly lutein, while chromoproteins consist of a phycoerythrin and two phycocyanins. Three papers (by D. B. Smith, A. N. O'Neill and A. S. Perlin; S. T. Bayley; and W. Yaphe), read by Dr. E. Gordon Young (Canada), were devoted to the structure and degradation of carragheenin. By fractionation with potassium chloride, this polysaccharide has been separated into a potassium-sensitive fraction $(K$ carragheenin) and a potassium-insensitive fraction ( $\lambda$-carragheenin); the former contains $3: 6$-anhydro$D$-galactose and sulphated $D$-galactose residues in nearly equal proportions, while the latter appears to be mainly a sulphated $D$-galactose polymer. It is interesting to note that this anhydro-sugar in carragheenin is the enantiomorph of that present in agar. Useful information on the structure of the two fractions has been obtained by $\mathrm{X}$-ray and infra-red studies. Bacteria capable of hydrolysing carragheenin and agar have been isolated from sea-water and marine algae; the cell-free enzyme solutions are specific for the respective polysaccharides, and have been used to identify agar and carragheenin in red algal extracts. Examination of the breakdown products should yield fruitful results.

Dr. Young, in a paper with D. L. Vincent and D. A. I. Goring, described the comparison of sodium 